\title{
Response of a sexually transmitted infection epidemic to a treatment and prevention programme in Nairobi, Kenya
}

\section{S Moses, E N Ngugi, A Costigan, C Kariuki, I Maclean, R C Brunham, F A Plummer}

Although it seems possible in a developing country context such as Kenya, given appropriate inputs and a sound approach, to shift a sexually transmitted disease (STI) epidemic from phase II to III, it is not entirely clear how to go beyond this stage, to low levels of endemicity or even elimination. Perhaps the most important challenge now is to expand STI treatment and community STI/HIV prevention programmes to a much larger scale. Although successful programmes have been implemented in many areas of sub-Saharan Africa on a small scale, a significant impact in reducing the STI/HIV burden will not occur until programme reach is expanded to district, provincial, and national levels.

See end of article for authors' affiliations

.................

Correspondence to: Dr. Stephen Moses Department of Medical Microbiology, University of Manitoba, 730 William Avenue, Fifth Floor, Winnipeg, Manitoba Canada R3E OW3; smoses@cc.umanitoba.ca

Accepted 30 November 2001
T he Republic of Kenya occupies approximately $580000 \mathrm{~km}^{2}$ in eastern Africa, bordered by Ethiopia, Somalia, Sudan, Tanzania, Uganda, and the Indian Ocean. There are seven provinces in the country and one administrative area (the capital city of Nairobi). Following a period of stagnant and at times negative economic growth in the early 1990s, economic performance in the mid-1990s improved, with annual growth in gross domestic product at about 4-5\%. Economic growth slowed again in the late 1990s, as political violence and social unrest adversely affected the important tourist industry, and the International Monetary Fund reduced support because of the slow pace of economic reform and alleged high levels of public sector corruption. ${ }^{1}$ In 1992 it was estimated that $42 \%$ of the population lived below the poverty line, and the unemployment rate in 1998 was estimated at 50\%.

The population of Kenya was estimated in 1999 to be just under 29 million, with a typical developing country population structure, including about $43 \%$ of the population under the age of 15. ${ }^{2}$ Population growth in 1999 was estimated at $1.6 \%$, with a birth rate of about $30 / 1000$ and a total fertility rate of 3.9. Infant mortality was estimated in 1999 at 59/1000 live births and average life expectancy at birth was 47 years. ${ }^{2}$ The majority of the population is rural, but the data in this paper draw principally from the capital city, Nairobi, with an estimated population of two million. More specifically, we focus on the vulnerable populations living in Nairobi's high density urban slums. These people or their parents or grandparents have generally migrated to Nairobi from rural homes in search of economic opportunities, and there is considerable population mobility, both within Nairobi, and back and forth to rural areas. ${ }^{3}$ As would be expected, people living in urban slums are poor, with low levels of education and limited opportunities for social and economic advancement. There are many distinct ethnic groups in Kenya, and they are all represented in Nairobi. There is evidence that the prevalence of HIV infection varies with ethnicity. ${ }^{4}$ Although national data on sexually transmitted infections (STIs) are lacking, data from the city of Mombasa on the Indian Ocean coast of Kenya and from Kisumu on Lake Victoria suggest that STI prevalences in Nairobi are comparable to those observed in other urban centres in Kenya. ${ }^{56}$

There are wide economic disparities within Kenya, and women are particularly disadvantaged in terms of social and economic opportunities. Health and other social services are in general poorly funded by government, and with the introduction of structural adjustment policies over the past decade, the situation has if anything worsened. The human development index (HDI) is an indicator that summarises the state of development of countries. It comprises measures of longevity (life expectancy at birth), knowledge (adult literacy and school enrolment), and standard of living (adjusted per capita income). Kenya's HDI rank in 1998 was 137 (of 174 nations), putting it among the group of low human development countries. ${ }^{7}$ As elaborated below, these social and economic circumstances enhance vulnerability to STIs and HIV infection.

\section{SEXUAL DYNAMICS}

The practice of women exchanging sex with men for money or gifts is common in Nairobi, and is largely home, bar, or lodging/brothel based. ${ }^{8}$ In a general population survey conducted in Kenya in 1989 as part of a World Health Organisation sponsored multi-country study examining sexual behaviour in several African countries, about 10\% of Kenyan men and 5\% of women reported engaging in commercial sex in the previous 12 months. ${ }^{9}$ The level of commercial sex was comparable to that reported from several other African countries. Although the sex trade is illegal and risky, in a climate where economic opportunities are limited (particularly for poor and less educated women), it offers advantages over existing alternatives: ease of entry, a ready market, and higher earnings than could be attained by other means. ${ }^{8}$ There is a wide variety of female sex workers, full time, part time, and casual, and 
women sometimes mix sex work with other economic activities, and move in and out of the sex trade over time. ${ }^{8}$ Male sex work is not common, although it may occur more often than appreciated. Clients of female sex workers include a wide variety of men from all social and occupational groups. Long distance truck drivers often travel through Nairobi and purchase sex from female sex workers, and are considered at particularly high risk of acquiring and transmitting STIs, including HIV infection. ${ }^{10}$ However, the clients of female sex workers are a very heterogeneous group. Both married and unmarried men often migrate to Nairobi from rural areas to find employment (leaving families behind) and commonly purchase sex from female sex workers. In a community survey we conducted in 1991/92 in a slum area of Nairobi, 14\% of men ( $38 \%$ of whom were married) reported purchasing sex in the previous three months, and they came from a wide variety of occupations and circumstances. ${ }^{11}$

Married men in Nairobi often have extramarital sex partners, married women much less commonly. In the same community survey in Nairobi, $15.7 \%$ of married men and 3.5\% of married women reported one or more extramarital sex partners in the previous three months. ${ }^{11}$ Extramarital or other concurrent partners may be commercial or non-commercial. The most important predictor of STI risk identified in the survey for both men and women was number of reported sex partners. ${ }^{11}$ In addition, and for men only, purchase of sex and being unmarried, or being married but living apart from their wives, were significant predictors of STI risk. Other sociodemographic and behavioural characteristics were not predictive of STI risk. The following paradigm was noted: most women, and married women in particular, had monogamous sexual partnerships; a relatively small number of women were active in the sex trade and had very many partners; and men played a bridging role between the core group of female sex workers and the lower risk women in the population. ${ }^{11}$

\section{HEALTH SERVICES}

Health services in Nairobi are offered in the public, private medical, and private informal sectors. Public sector STI related services are provided primarily by the Nairobi City Council Health Department, and comprise health centres (which provide both curative and preventive services), maternity units, and a maternity hospital. The private informal sector is readily accessible and often used, but services provided are usually of poor quality. ${ }^{12}$ User charges have been introduced in recent years for public sector health services, and these may have had an adverse impact on service accessibility. ${ }^{13}$

The economically marginalised populations which are the subject of the intervention programme described below most often use the public and private informal sectors, as the private medical sector is usually too costly to access. Laboratory testing is generally unavailable in these contexts.

A survey that we conducted in 1991 of STI patients at several health centres in Nairobi and the town of Nakuru in western Kenya indicated that about one quarter of patients with STI related symptoms waited longer than two weeks before seeking care, and women waited significantly longer than men. ${ }^{12}$ Accessibility to services is often problematic for a variety of reasons, particularly for women. Barriers to access for women were felt to include time constraints (owing to the burden of household duties), lack of financial resources to cover transportation and other costs involved in attending a health clinic, and fear of stigmatisation. ${ }^{12}$

\section{SEXUALLY TRANSMITTED INFECTIONS}

The prevalence of sexually transmitted infections in the general adult population of Kenya is unknown, but information is available from studies on specific populations. In a study of unselected antenatal clinic attenders in Nairobi in 1994, the prevalences of syphilis, gonorrhoea, chlamydial infection, and trichomoniasis were found to be $3.1 \%, 2.4 \%, 8.8 \%$, and $19.9 \%$, respectively. ${ }^{14}$ This compares reasonably closely to the prevalences estimated by Gerbase et al for women in all countries in sub-Saharan Africa in 1995 (3.9\%, 2.8\%, 7.1\%, and $14.1 \%$, respectively). ${ }^{15}$ In a study of male trucking company employees (including long distance truck drivers) in the Kenyan port city of Mombasa in 1994-95, the prevalences of the same four STIs were $5.3 \%, 3.4 \%, 3.6 \%$, and $6.0 \%$, respectively. ${ }^{16}$ This also compares reasonably closely with Gerbase's STI prevalence estimates for men in sub-Saharan Africa (3.1\%, 2.0\%, 4.8\%, and $1.4 \%$ ). The generally higher rates seen among the Mombasa men may reflect their higher risk for STIs than the general population of men. The somewhat lower rate for chlamydial infection observed in Mombasa may reflect the use of a relatively low sensitivity test in that study (enzyme immunoassay). In any case, these data overall are consistent with the notion that the STI risk in the general population of Kenya is similar in magnitude to that of other countries in sub-Saharan Africa, and for most STIs this is an order of magnitude higher than that observed in other regions of the world. ${ }^{15}$ Although population based data before 1990 are lacking, it would seem that the STI epidemic in Nairobi in the early 1990s (and probably before that period) was in an early hyperendemic phase, or phase II according to the dynamic topology model of STI epidemics developed by Wasserheit and Aral. ${ }^{17}$ In this model, an STI epidemic is characterised as evolving through predictable phases, determined by the interaction among the pathogen, the population in which it is found, and prevention efforts that are developed to control it. Phase II is characterised by generally high STI prevalence and limited prevention efforts.

\section{DESCRIPTION OF THE STI TREATMENT AND PREVENTION PROGRAMME}

Up to the beginning of the 1990s, STI related public sector health services in Nairobi were provided largely through a centralised STI clinic called the special treatment clinic (STC). All individuals presenting to public sector primary health care centres in Nairobi with STI related symptoms were referred there for assessment and treatment, and in addition many patients presented directly to the STC for primary care. Attempts were made to make disease specific diagnoses, but without adequate laboratory support to do so accurately. There were major problems associated with this system, including long waiting times, poor quality of services, lack of accessibility, limited attention to counselling and prevention, and stigmatisation. Beginning in 1991, with support from the Governments of Canada and Belgium and others, the Nairobi City Council embarked on a programme to decentralise STI services to the primary health care level, using a syndromic approach to case management. Health workers, supervisors, and trainers were trained, a supervisory system put into place, the STI drug supply was assured, and a system established for referrals. Through additional health worker training, the provision of STI/HIV related education and counselling services were strengthened, emphasising condom promotion in particular. Syphilis screening services were also decentralised, with syphilis testing facilities established at local clinics. ${ }^{18}$ Efforts have been made to enhance partner notification, but have had limited success except in the context of antenatal women with syphilis. ${ }^{19}$

At the same time, community STI/HIV related prevention efforts in the communities served by the health facilities were initiated, directed primarily at high risk groups such as female sex workers, but also at high risk men (such as long distance truck drivers) and youths, both in and out of school. These programmes have generally taken the form of peer mediated education programmes involving trained peer educators, and also supervisors and trainers from the Nairobi City Council. ${ }^{8}$ Using a peer mediated approach, information is provided to 
female sex workers and other high risk individuals regarding prevention of STIs and HIV infection, the importance of consistent and correct condom use, and the need for prompt treatment of STIs. Condoms are distributed at no charge through the peer educators and through health centres. The programme now comprises five Nairobi City Council health centres in two distinct parts of the city and the communities that they serve, with a catchment population of approximately 400 000. Activities have also been expanded to include other health centres and communities in Nairobi, as well as the towns of Nakuru and Thika, and more recently communities in western Kenya. Community non-governmental organisations have been trained to undertake both clinic and community based STI treatment and prevention programmes, based on the same models.

After approximately seven years of operation in Nairobi, the following outputs have been achieved in relation to the facility based component of the programme: accessible STI/HIV related health services of high quality are available at programme clinics, with high levels of service utilisation and condom distribution; STI management algorithms have been developed, tested and refined; training courses in STI management, clinic supervision, and training of trainers have been developed, tested, and refined; systems for record keeping and referral have been developed; over 500 health workers and 50 trainers/supervisors have been trained directly in STI management, and many others indirectly through nongovernmental organisations and other implementers (inservice training); and over 100 tutors and 25 trainers have been trained through the Kenya Medical Training College (pre-service training), with the syndromic approach to STI management now forming part of its core curriculum.

The following outputs have been achieved in relation to the community based component of the programme: peer mediated education programmes directed at high risk groups have been developed at programme sites, involving approximately 2000 female sex workers and a similar number of high risk men; training courses for peer educators, supervisors, and trainers have been developed, tested, and refined; over 100 peer educators have been trained and are active in the community; and over 50000 condoms are being distributed monthly to the high risk groups indicated above.

\section{MONITORING PROGRAMME IMPACT: BEHAVIOURAL AND STI SURVEILLANCE}

Several surveillance methods have been used to monitor programme impact at the population level during the period 1992-1999. These include:

- Behavioural surveillance through serial surveys among representative antenatal clinic attenders, 1992-1999.

- STI surveillance through serial surveys among representative antenatal clinic attenders, 1992-1999.

- Monitoring of attendance patterns for STI syndromes at programme area health centres.

The behavioural and STI surveillance of antenatal clinic attenders was conducted in three of the Nairobi City Council health centres where STI service provision was enhanced through the programme. Their catchment area, with a population of approximately 200000 people, comprises one of the programme's communities where STI/HIV prevention efforts were initiated. Between April 1992 and December 1999, six survey rounds were completed as follows:

Survey 1: March 1992 - September 1992

Survey 2: October 1992 - October 1993

Survey 3: November 1993 - November 1994

Survey 4: December 1994 - March 1996
Survey 5: May 1996 - April 1997

Survey 6: February 1999 - December 1999

During each round, the first five antenatal women presenting to a clinic each day were recruited. Each clinic was revisited daily until approximately 300 women were recruited, which generally took two to three months. The three clinics were visited in sequence. When a given survey round was completed at one clinic, data collection at the next one began. Over $95 \%$ of women identified agreed to participate. A standardised questionnaire, which collected basic sociodemographic information and information on sexual behaviour, condom knowledge and use, and history of STIs, was administered. All women received HIV pretest counselling from a trained nurse after informed consent had been obtained. A $10 \mathrm{ml}$ sample of blood was drawn and transported to the laboratory at the department of medical microbiology of the University of Nairobi.

Serum samples were tested for HIV-1 using a standard enzyme immunoassay (EIA) technology (Detect HIV 1/2, Biochem Immunosystems, Montreal, Canada). Positive samples were confirmed for HIV-1 using a recombinant EIA system (Recombigen HIV 1/2, Trinity Biotech, Galway, Ireland). Syphilis seropositivity was determined using the rapid plasma reagin (RPR) test (Macro-Vue RPR Card Tests, Becton Dickinson International, Meylan, France) and confirmed by a Treponema pallidum haemagglutination test (TPHA Test Kits, Biotec Laboratories, Ipswich, UK). Cervical swab specimens were plated on modified Thayer-Martin media for $\mathrm{N}$ gonorrhoeae culture. Results were conveyed to the women along with HIV post-test counselling at a follow up visit one week later, and specific STI treatment was provided where indicated, according to the Kenya National AIDS and STD Control Programme (NASCOP) guidelines. Aliquots of serum were frozen and shipped to the laboratory at the department of medical microbiology, University of Manitoba, in Winnipeg, Canada, where they were tested for antibody to $C$ trachomatis using an enzyme immunoassay technique based on antibodies to serovars L2, E, F, and K. Each specimen was run in duplicate along with two different negative controls, and the result was considered positive if the mean optical density of the specimen was more than three times the mean optical density of the controls.

Since 1992, health centre staff have kept records of patients presenting with STI related syndromes, stratified by age and sex. The syndromes include urethral discharge in men, vaginal discharge or pruritus in women, genital ulcer disease, pelvic inflammatory disease, and ophthalmia neonatorum. To examine trends in clinic attendance for these syndromes over time, records were tabulated for the period 1992-1998, as were records of overall clinic attendance.

Data were analysed principally using SPSS for Windows, version 10.0. Proportions were compared using the $\chi^{2}$ test and trends were evaluated using the $\chi^{2}$ test for trend. Multiple logistic regression models were used to examine the effect of age and ethnic origin in determining STI/HIV prevalence and behavioural outcomes. Poisson regression using SAS, version 8.0, was used to evaluate trends in clinic attendance for STI syndromes over time.

\section{RESULTS OF BEHAVIOURAL AND STI SURVEILLANCE}

In all, 5459 women were sampled over the survey period, approximately 300 women per clinic per survey. The mean age was 23.2 years (range 13 to 48 ), and $84.5 \%$ of the women were married, $13.5 \%$ were never married, $1.8 \%$ were separated or divorced, and $0.3 \%$ were widowed. The age distribution of the population is shown in table 1 . Exchange of sex for money or gifts (ever) was reported by $6.2 \%$ of women and $3.3 \%$ reported doing so in the previous three months. As shown in table 1, women reported relatively few sex partners, both lifetime and in the previous three months. 


\begin{tabular}{|c|c|c|}
\hline Characteristic & $\mathrm{n}$ or mean & Per cent or range \\
\hline Age (years) & 23.2 & $13-48$ \\
\hline \multicolumn{3}{|l|}{ Age category } \\
\hline$<20$ & 1153 & 21.1 \\
\hline $20-24$ & 2435 & 44.6 \\
\hline $25-29$ & 1323 & 24.2 \\
\hline $30-34$ & 414 & 7.6 \\
\hline$\geqslant 35$ & 132 & 2.4 \\
\hline \multicolumn{3}{|l|}{ Province of origin ${ }^{4}$} \\
\hline Central/eastern group & 2236 & 41.0 \\
\hline Western group & 1181 & 21.7 \\
\hline Nyanza & 2035 & 37.3 \\
\hline \multicolumn{3}{|l|}{ Marital status } \\
\hline Married & 4610 & 84.5 \\
\hline Never married & 734 & 13.5 \\
\hline Separated/divorced & 97 & 1.8 \\
\hline Widowed & 16 & 0.3 \\
\hline \multicolumn{3}{|c|}{ Reported sex partners, lifetime } \\
\hline 0 & 0 & 0 \\
\hline 1 & 1391 & 25.5 \\
\hline $2-5$ & 3882 & 71.1 \\
\hline$\geqslant 6$ & 179 & 3.3 \\
\hline \multicolumn{3}{|c|}{ Reported sex partners, previous three months } \\
\hline 0 & 267 & 4.9 \\
\hline 1 & 4983 & 91.3 \\
\hline $2-5$ & 173 & 3.2 \\
\hline$\geqslant 6$ & 22 & 0.4 \\
\hline \multicolumn{3}{|c|}{ Exchange of sex for money or gifts } \\
\hline Ever & 337 & 6.2 \\
\hline Previous three months & 179 & 3.3 \\
\hline
\end{tabular}

Several significant trends in sexual behaviour emerged over time. First, as shown in table 2, there were statistically significant declines in the proportion of women reporting receiving money or gifts for sex work, both lifetime and in the previous three months. There were also significant declines in the reported occurrence of sexually transmitted infections (both ever and in the previous three months) among women reporting sex work.

It should be noted that in survey 6 there were only 12 women reporting sex work, so the rate is unstable. Among women reporting no history of sex work, there were statistically significant declines in both reported lifetime sex partners and new sex partners in the previous three months. However, a recent upward trend was observed, particularly in survey 6, which is of concern. There was a statistically significant trend towards a reduction in perception of AIDS risk, which is largely related to a decreasing trend in believing that the husband or other main partner had other partners. This is the only evidence that we have, albeit indirect, of potential changes over time in male sexual behaviour. Although reported ever use of condoms increased over time and reported recent condom use increased slightly, condom use remained very low throughout the time period, particularly for recent use. However, there is evidence that condom use among women involved in the sex trade increased substantially. In surveys conducted among over 700 female sex workers residing and working in the catchment area of the health centres in 1994 and 1998, the proportion of women reporting never using condoms declined from $43 \%$ to virtually zero, and female sex workers reporting always using condoms increased from $22 \%$ to $86 \%$ ( $p<0.001$, Plummer FA, unpublished data).

The changes observed towards safer sexual behaviour are reflected in STI prevalence over time in the antenatal populations surveyed. Table 3 shows declines in rates of gonorrhoea and syphilis, from 5.3\% and 5.4\%, respectively, in 1992 to $1.7 \%$ and $2.5 \%$ in 1999 . Both declines were statistically significant. These are acute STIs, with relatively short durations of infection, so serial prevalence rates should be reasonable approximations to incidence. Although chlamydia is also an acute infection, we measured chlamydia antibody in serum, which is long lived, even after treatment. Therefore, as for HIV, we also examined chlamydial prevalence in women under 20 , as this is likely to be a closer approximation to incidence. For chlamydia, prevalence rates declined from almost 33\% in 1992 to $17 \%$ in $1999(\mathrm{p}<0.001)$. The pattern for women under 20 was virtually identical. For HIV infection, overall prevalence rates stabilised at about 18\% between 1993 and 1999 (surveys 2 to 6), but rates among women under 20, after increasing from about $15 \%$ in 1992 to a high of about $21 \%$ in 1993 and 1994 (surveys 2 and 3), subsequently declined to $14 \%$ in 1999 (survey 6).

As age and ethnicity may be associated with HIV risk ${ }^{4}$ (and perhaps STI risk and behavioural outcomes), the trends over time described above for behavioural parameters and STI prevalence were examined in multiple logistic regression models, with STIs and behavioural outcomes as outcome variables, and age, province of origin, and survey round as independent variables. Controlling for age and province of origin in this way did not affect the statistical significance of any of the trends reported above. As has been shown previously, ${ }^{4}$ HIV prevalence (but not the other STIs measured) was significantly higher among women from Nyanza province than in those from other provinces $(p<0.001)$. In addition, reported ever use of condoms was higher among women over 20 than under $20(\mathrm{p}=0.004)$, and exchange of money for sex or gifts was more common among women under 20 than over $20(\mathrm{p}<0.001)$.

After STI care was introduced into the health centres in 1991 and 1992, STI attendance increased rapidly, as would be expected, peaking in 1993 for all syndromes (fig 1A). Attendance declined in subsequent years, through 1998, and all

Table 2 Trends over time in reported sexual behaviour, perception of AIDS risk, perception of partner behaviour, and reported condom use among antenatal clinic attenders screened in Nairobi, 1992-1999, n=5459

\begin{tabular}{|c|c|c|c|c|c|c|c|}
\hline & Survey 1 & Survey 2 & Survey 3 & Survey 4 & Survey 5 & Survey 6 & p Value* \\
\hline Receipt of money or gifts for sex, lifetime & $9.2 \%$ & $9.8 \%$ & $6.5 \%$ & $4.8 \%$ & $5.3 \%$ & $1.3 \%$ & $<0.001$ \\
\hline Receipt of money or gifts for sex, previous 3 months & $4.5 \%$ & $5.3 \%$ & $3.6 \%$ & $2.0 \%$ & $3.0 \%$ & $1.2 \%$ & $<0.001$ \\
\hline Reported STIs among female sex workers, lifetime & $38.5 \%$ & $43.6 \%$ & $39.3 \%$ & $27.3 \%$ & $12.5 \%$ & $33.3 \%$ & 0.003 \\
\hline Reported STIs among female sex workers, previous 3 months & $24.4 \%$ & $23.4 \%$ & $24.6 \%$ & $11.4 \%$ & $4.2 \%$ & $25.0 \%$ & 0.01 \\
\hline More than 5 lifetime sex partners among non-sex-workers & $3.9 \%$ & $3.0 \%$ & $1.8 \%$ & $2.1 \%$ & $0.8 \%$ & $2.3 \%$ & 0.001 \\
\hline $\begin{array}{l}\text { More than } 2 \text { sex partners in previous } 3 \text { months among } \\
\text { non-sex workers }\end{array}$ & $1.0 \%$ & $3.9 \%$ & $2.3 \%$ & $0.6 \%$ & $1.0 \%$ & $1.4 \%$ & 0.01 \\
\hline Perceives herself to be at risk for AIDS & $39.4 \%$ & $37.3 \%$ & $34.5 \%$ & $23.6 \%$ & $24.7 \%$ & $21.2 \%$ & 0.001 \\
\hline Perceives that partner has other partners & $29.5 \%$ & $27.5 \%$ & $19.7 \%$ & $23.5 \%$ & $20.8 \%$ & $11.2 \%$ & 0.001 \\
\hline Has ever used condoms & $2.5 \%$ & $6.5 \%$ & $2.4 \%$ & $7.7 \%$ & $6.8 \%$ & $19.9 \%$ & 0.001 \\
\hline Has used condoms in previous 3 months & $0.4 \%$ & $2.1 \%$ & $0.7 \%$ & $1.6 \%$ & $1.1 \%$ & $2.5 \%$ & 0.11 \\
\hline
\end{tabular}


Table 3 Trends over time in sexually transmitted infections detected among antenatal clinic attenders screened in Nairobi, 1992-1999, $n=5459$

\begin{tabular}{|c|c|c|c|c|c|c|c|}
\hline & Survey 1 & Survey 2 & Survey 3 & Survey 4 & Survey 5 & Survey 6 & p Value* \\
\hline Gonorrhoea (cervical swab) & $5.3 \%$ & $5.0 \%$ & $3.6 \%$ & $3.5 \%$ & $3.9 \%$ & $1.7 \%$ & $<0.001$ \\
\hline Syphilis (serology) & $5.4 \%$ & $5.2 \%$ & $4.4 \%$ & $3.9 \%$ & $3.7 \%$ & $2.5 \%$ & $<0.001$ \\
\hline Chlamydial infection (serology), all women & $32.9^{*}$ & $30.8 \%$ & $20.5 \%$ & $22.5 \%$ & $\dagger$ & $17.0 \%$ & $<0.001$ \\
\hline Chlamydial infection (serology), women under age 20 & $34.3 \%$ & $29.3 \%$ & $18.0 \%$ & $19.4 \%$ & $\dagger$ & $17.0 \%$ & $<0.001$ \\
\hline HIV infection (serology), all women & $14.7 \%$ & $19.0 \%$ & $17.9 \%$ & $17.5 \%$ & $18.0 \%$ & $18.7 \%$ & Not significant \\
\hline HIV infection (serology), women under age 20 & $15.0 \%$ & $20.8 \%$ & $20.8 \%$ & $14.4 \%$ & $15.1 \%$ & $14.1 \%$ & 0.03 , Surveys 2-6 \\
\hline
\end{tabular}

declines were statistically significant. It is of course possible that these declines may have reflected changes in health care seeking behaviour, rather than changes in disease incidence. For example, patients with STI symptoms in later years may have preferentially accessed the private or informal health sectors. There is no evidence, however, that this occurred, and the private sector has remained a much more expensive and less accessible option for most patients than the public sector. There is also evidence of general satisfaction among STI patients, with the enhanced care being offered in public sector health centres strengthened through the intervention programme. ${ }^{20}$ As indicated previously, it is possible that the imposition of user fees may have deterred individuals from seeking care at public sector health centres, but overall attendance at health centres has remained relatively constant
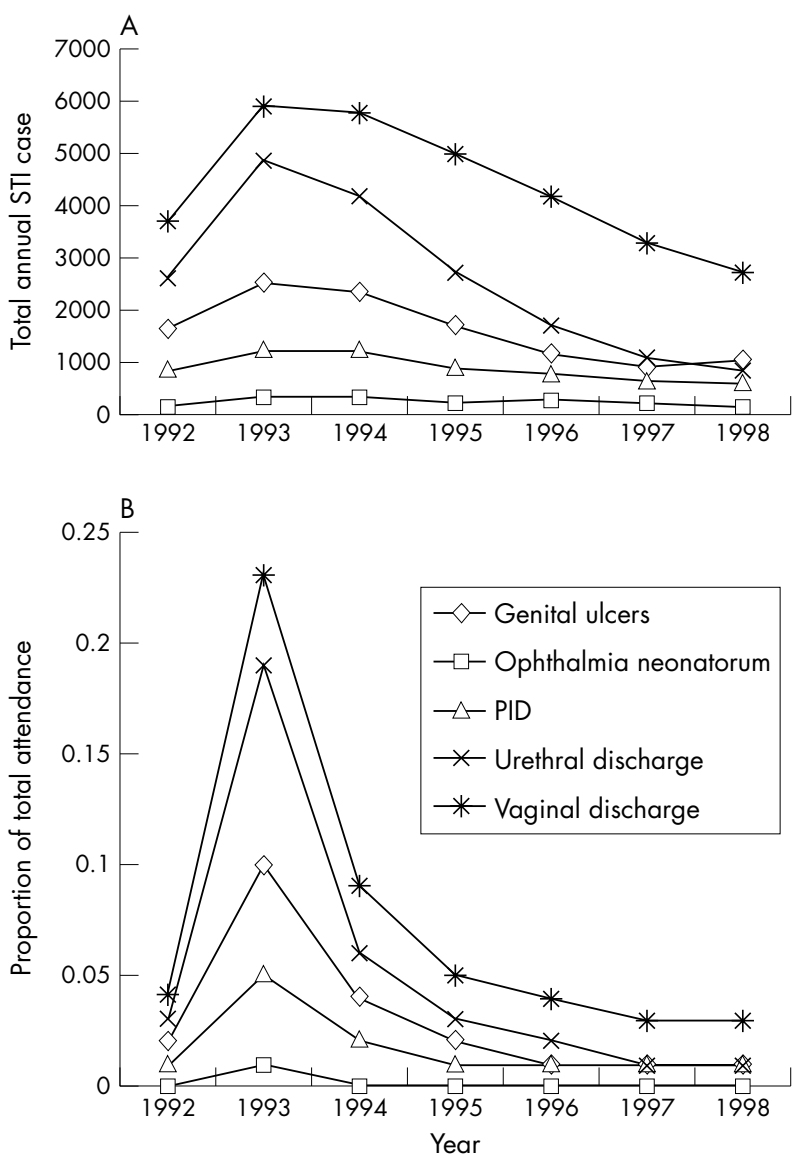

Figure 1 (A) Trends over time in attendance at Nairobi project clinics for STI syndromes, 1992-98. Declining trends for all syndromes, $p<0.001$ (Poisson regression). (B) Trends over time in attendance at Nairobi project clinics for STI syndromes as a proportion of all clinic attendance, 1992-98. Declining trends for all syndromes, $p<0.001$ ( $\chi^{2}$ test for trend). PID, pelvic inflammatory disease; STI, sexually transmitted infection. over the period under examination. As shown in fig $1 \mathrm{~B}$, STI attendance as a proportion of overall attendance shows a pattern over time that is very similar to that seen with overall attendance, peaking for all syndromes in 1993, then falling steadily thereafter.

\section{DISCUSSION}

Nairobi-like most major centres in sub-Saharan Africa in the 1980s and 1990s - has been experiencing a largely uncontrolled STI epidemic that has been in a hyperendemic phase (phase II) of its development according to the classification of Wasserheit and Aral. ${ }^{17}$ To make inroads into the epidemic, the intervention developed in Nairobi consisted of providing effective, non-stigmatising STI/HIV related treatment and counselling services to the general population at their first point of contact with the health system, and at the same time preventing STI/HIV transmission by promoting safer sex practices and improved health care seeking behaviour among high risk groups in the community. The intervention programme thus included several of the key STI prevention strategies proposed by Wasserheit and Aral for phase II declines in STI epidemics: detection and treatment services, provider risk reduction counselling, and client initiated partner notification (directed primarily at "maintenance networks"); and targeted health promotion, outreach for screening and treatment, and peer risk reduction counselling (directed primarily at "spread networks"). In a relatively short period of time, declines in STI prevalence among sentinel antenatal clinic attenders and safer sex practices among female sex workers in the community could be documented. This appears to have had the effect of moving the epidemic from a hyperendemic to a decline phase (phase III), ${ }^{17}$ at least in the areas under observation. As the two major programme elements (STI treatment and targeted community prevention) were integrated, it is not possible to disentangle their individual effects. However, as noted by Wasserheit and Aral, a two pronged strategy which initiates STI prevention approaches simultaneously in maintenance and spread networks should result in more rapid and sustained declines in STI incidence and morbidity. ${ }^{17}$ In the Wasserheit-Aral model, initial declines in STI prevalence occur in lower risk populations as STI prevention measures are mounted, eventually concentrating the burden of infection in more difficult to reach core groups. However, in Nairobi, there is evidence for simultaneous declines in prevalence in both low risk and high risk populations, presumably because interventions were directed specifically and preferentially to higher risk groups, particularly to female sex workers.

In most developing country settings, routine STI reporting is of little value for surveillance purposes because ascertainment is so incomplete. Health care providers, whether in the private or public sectors, generally do not report STI cases systematically to health departments. Furthermore, STIs are often managed in the informal sector or go unrecognised and untreated for long periods of time. Sentinel STI surveillance among groups which are representative of the general population is therefore an approach that can be used to monitor 
trends. Antenatal women are one such group. They have the advantage of being relatively easy to access, and in urban centres, the majority of antenatal women attend a health centre for at least one antenatal visit, so coverage is high. They may not be entirely representative of the general adult female population (it has been shown, for example, that HIV seroprevalence among antenatal women may underestimate true seroprevalence ${ }^{21}$ ), but accessing more representative groups of women (or men for that matter) requires more complex and much more costly sampling techniques. Antenatal women can be a reasonable surrogate for monitoring behavioural and STI trends in the general population in many settings.

Although it is not possible to attribute the observed reductions in STI rates entirely to programme activities, it is likely that they played a major role. It would be expected that good quality, accessible STI treatment services, with wide coverage, should reduce STI transmission by shortening the duration of STI infectivity, one of the key variables determining the basic reproductive rate of STI epidemics. ${ }^{22}$ Similarly, increased condom use and other safer sex practices on the part of female sex workers would be expected to have a major impact on reducing STI transmission because of the important role played by these groups in STI spread ${ }^{24}$ Reductions in numbers of sex partners were reported over time by women in the community, and fewer women reported engaging in sex work. In addition, they perceived that their male partners were less likely to have other partners. Such behavioural change in the general population was not a specific focus of the intervention programme (except for those women presenting to health centres with STI related symptoms) and may have resulted from government media campaigns or other health education efforts, or simply from emerging awareness of the HIV/AIDS epidemic, as has been reported in Uganda and linked to declines in HIV infection. ${ }^{25}$ It is difficult to attribute behaviour change to a specific event or phenomenon. In Nairobi, however, unlike in Uganda, condom use by women in the general community has remained at relatively low levels. This is consistent with data from the most recent Kenya Demographic and Health Survey, which indicated that in 1998, condom use by both men and women in the general population was quite low: overall $6 \%$ of women and $21 \%$ of men reported condom use during their last sexual intercourse. ${ }^{26}$ Increased condom use in the general population therefore does not seem to be a major contributing factor to the declines in STI rates.

Although establishing effective, accessible, affordable, and decentralised public sector services for STI management is achievable, it is not necessarily an easy task. Our experience with the intervention programme in Nairobi identified a number of constraints that needed to be confronted and overcome. These included:

- Inadequate basic training and inefficient deployment of health workers.

- Stigmatising attitudes of health workers towards marginalised groups such as female sex workers.

- High patient loads at health centres.

- Lack of supportive supervision.

- Inadequate referral systems.

- Chronic shortages of clinic supplies and drugs for STI treatment.

- Inadequate recording of health information.

Baseline surveys among health workers and qualitative assessments of health centre staff and operations were very helpful in designing and developing the intervention programme. Participatory training programmes for health workers both increased skill levels and helped to counteract negative attitudes. We learned many important lessons in programme implementation, in both the facility based and community based components:

- A syndromic approach to STI management is effective and is acceptable to both STI patients and health care providers.

- Sustainability must be a consideration from the beginning. It requires integration of what is new into existing structures, ownership, and involvement by local managers, joint planning and decision making, and local capacities to supervise and train.

- Training should be practical, participatory, and realistic, focusing on the tasks that trainees will perform. By the end of training, participants should understand what new tasks are expected of them and should have the necessary skills to perform them.

- Trainers must acquire the necessary skills to modify training as well as deliver it.

- High quality supervision of both health workers and peer educators is essential, so that continuing skills development and upgrading forms an integral part of their work. Supervisory capacity needs to be built into all peripheral points of implementation, in addition to central levels.

- Developing health worker skills in STI/HIV counselling, as well as in condom promotion and demonstration, pose the greatest difficulties and require the most attention.

- With initial education and support, female sex workers can organise themselves into dynamic self help collectives. Peer educators are key to group cohesion and support, and are critical in promoting attitude and behaviour change.

Program sustainability is an important goal, and the decentralised STI management programme developed in Nairobi has now largely been devolved to the local authorities. However, given the current economic situation in Kenya, as in most countries in sub-Saharan Africa, it is not realistic to expect that the costs of drugs and other commodities (including condoms) can be borne solely by local governments. Furthermore, as has been indicated previously, passing on these costs to clients is likely to deter them from seeking care. In Kenya, the cost of STI drugs and condom provision in the public sector is largely supported by the World Bank and other donor agencies. In the short to medium term, external donor agencies have an important role to play in underwriting such expenditures. Sustainability of other elements of programme delivery is an achievable objective and it is a continuing challenge to ensure that high quality programmes are maintained within local structures.

Although it seems possible in a developing country context such as Kenya, given appropriate inputs and a sound approach, to shift an STI epidemic from phase II to III, ${ }^{17}$ it is not entirely clear how to go beyond this stage, to low levels of endemicity or even elimination. Perhaps the most important challenge now is to expand STI treatment and community STI/HIV prevention programmes to a much larger scale. Although successful programmes have been implemented in many areas of sub-Saharan Africa on a small scale, a significant impact in reducing the STI/HIV burden will not occur until programme reach is expanded to district, provincial, and national levels. ${ }^{8}$ This will require substantial in-country political support and leadership, as well as external support from development assistance agencies. As inroads are made into the STI burden through expanded treatment services, the epidemic will concentrate further among high risk, marginalised populations. Thus continued and perhaps increased emphasis on core groups will be required as the epidemic evolves. Continuing surveillance is required to evaluate programme effectiveness, and ongoing applied research is important for a better understanding of programme achievements and limitations. 


\section{ACKNOWLEDGEMENTS}

This work was supported by a grant from the Canadian International Development Agency (Project "Strengthening STD/AIDS Control in Kenya", No 524/19147). FAP is the recipient of Senior Scientist award, Canadian Institutes Health Research. SM is the recipient of an Investigator award, Canadian Institutes of Health Research. We wish to thank the staff of the Project and of the Nairobi City Council Health Department for their continuing cooperation, hard work and support.

\section{Authors' affiliations}

S Moses, F A Plummer, Departments of Medical Microbiology, Community Health Sciences and Medicine, University of Manitoba,

Winnipeg, Canada

E N Ngugi, A Costigan, C Kariuki, Department of Community Health, University of Nairobi, Nairobi, Kenya

I Maclean, Department of Medical Microbiology, University of Manitoba, Winnipeg, Canada

R C Brunham, British Columbia Centre for Disease Control, University of British Columbia, Vancouver, Canada

\section{REFERENCES}

1 United States Department of State. Background Notes: Republic of Kenya, March 1998. http://www.state.gov/www/background_notes.

2 United States Central Intelligence Agency. The World Factbook 1999 - Kenya. http://www.ocdi.gov/cia/publications/factbook.

3 Ferguson AG. Fertility and contraception adoption and discontinuation in rural Kenya. Stud Fam Plann 1992;23:257-67.

4 Jackson DJ, Ngugi EN, Plummer FA, et al. Stable antenatal HIV-1 seroprevalence with high population mobility and marked seroprevalence variation among sentinel sites within Nairobi, Kenya. AIDS 1999:13:583-90.

5 Jackson DJ, Rakwar JP, Richardson BA, et al. Decreased incidence of sexually transmitted diseases among trucking company workers in Kenya: results of a behavioural risk-reduction programme. AIDS 1997:11:903-9.

6 Buve A, Weiss HA, Laga M, et al. The epidemiology of gonorrhoea, chlamydial infection and syphilis in four African cities. AIDS 2001;5(suppl 4):S79-88

7 United Nations Development Programme. Human Development Report 1998. New York: Oxford University Press, 1998.

8 Ngugi EN, Wilson D, Sebstad J, et al. Focused peer-mediated educational programs among female sex workers to reduce sexually transmitted disease and human immunodeficiency virus transmission in Kenya and Zimbabwe. J Infect Dis 1996;174(suppl 2):S240-7.

9 Carael M, Cleland J, Deheneffe JC, et al. Sexual behaviour in developing countries: implications for HIV control. AIDS 1995; 9:1171-5.
10 Bwayo J, Plummer F, Omari $M$, et al. Human immunodeficiency infection in long-distance truck drivers in east Africa. Arch Intern Med 1994;154:1391-6.

11 Moses S, Muia E, Bradley JE, et al. Sexual behaviour in Kenya: implications for sexually transmitted disease transmission and control. Soc Sci Med 1994:39:1649-56.

12 Moses S, Ngugi EN, Bradley JE, et al. Health care-seeking behavior related to the transmission of sexually transmitted diseases in Kenya. Am J Public Health 1994;84:1947-51.

13 Moses S, Manji F, Bradley JE, et al. Impact of user fees on attendance at a referral centre for sexually transmitted diseases in Kenya. Lancet 1992:340:463-6.

14 Thomas T, Choudhri S, Kariuki C, Moses S. Identifying cervical infection among pregnant women in Nairobi, Kenya: limitations of risk assessmen and symptom-based approaches. Genitourin Med 1996;72:334-8.

15 Gerbase AC, Rowley JT, Heymann DHL, et al. Global prevalence and incidence estimates of selected curable STDs. Sex Transm Inf 1998;74(suppl 1):S12-16

16 Jackson DJ, Rakwar JP, Chohan B, et al. Urethral infection in a workplace population of East African men: evaluation of strategies for screening and management. J Infect Dis 1997;175:833-8.

17 Wasserheit JN, Aral SO. The dynamic topology of sexually transmitted disease epidemics: implications for prevention strategies. J Infect Dis 1996; 174(suppl 2):S201-13.

18 Jenniskens F, Obwaka E, Kirisuah S, et al. Syphilis control in pregnancy: decentralization of screening facilities to primary care level, a demonstration project in Nairobi, Kenya. Int J Gynaecol Obstet 1995;8(suppl): S121-8.

19 Nieru EK, Eldridge G, Ngugi EN, et al. STD partner notification and referral in primary level health centres in Nairobi, Kenya. Sex Transm Dis 1995;22:231-5.

20 Moses S, Bradley JE, Muia E, et al. Evaluation of an in-service training programme in STD management for clinical officers and nurses in Kenya [abstract]. In: Final Program and Abstract Book, VIII International Conference on AIDS in Africa, Yaounde, Cameroon, December 8-11, 1992

21 Gray RH, Wawer M, Serwadda D, et al. Population-based study of fertility in women with HIV-1 infection in Uganda. Lancet 1998;351:98-103

22 Yorke JA, Hethcote HW, Nold A. Dynamics and control of the transmission of gonorrhea. Sex Transm Dis 1978:5:51-6.

23 Anderson RM, May RM. Epidemiologic parameters of HIV transmission. Nature 1988;333:514-19.

24 Plummer FA, Nagelkerke NJD, Moses S, et al. The importance of core groups in the epidemiology and control of HIV-1 infection. AIDS 1991:5(suppl 1):S169-76

25 Asiimwe-Okiror G, Opio AA, Musinguzi J, et al. Change in sexual behaviour and decline in HIV infection among pregnant women in urban Uganda. AIDS 1997;11:1757-63

26 Kenya Demographic and Health Survey 1998. Preliminary report. Demographic and health surveys. Calverton, Maryland: Macro International Inc, 1998:13. 